\title{
Research Paper: The Effectiveness of Mindfulness Training on Emotional Schemas Mothers of Children With Cerebral Palsy
}

\section{Nasim Aghdasi $\left.{ }^{1} \mathbb{(}\right),{ }^{*}$ Ali Akbar Soleimanian ${ }^{1} \mathbb{C}$, Roghayeh Asadi Gandomani ${ }^{2}$}

1. Department of Counseling, Guidance and Education, Faculty of Humanities, University of Bojnord, Bojnord, Iran 2. Department of Psychology, Faculty of Humanities, University of Bojnord, Bojnord, Iran.

\begin{tabular}{l|l}
$\begin{array}{l}\text { Use yourdevice toscan } \\
\text { and read the article online }\end{array}$ & $\begin{array}{l}\text { Cftatton Aghdasi N, Soleimanian AA, Asadi Gandomani R. [The Effectiveness of Mindfulness Training on Emotional } \\
\text { Schemas Mothers of Children With Cerebral Palsy (Persian)]. Archives of Rehabilitation. 2019; 20(1):86-97. http://dx.doi. } \\
\text { org/10.32598/rj.20.1.86 }\end{array}$ \\
dol http://dx.doi.org/10.32598/rj.20.1.86
\end{tabular}

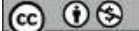

Received: 28 Aug 2018 Accepted: 13 Jan 2019 Available Online: 01 Apr 2019

Keywords:

Mindfulness,

Emotional schemas,

Cerebral Palsy

\section{ABSTRACT}

Objective Mothers of children with Cerebral Palsy (CP) are susceptible to emotional problems like stress, anxiety, and depression which can lead to emotional schemas like rumination, not expressing emotions, lack of values, and feeling guilty. These negative beliefs about emotions cause ineffective thoughts and inappropriate behavior patterns that affect the mental health of the mothers. In this case, interventions such as mindfulness education can be effective in reducing their maladaptive emotional schemas by adjusting attention, developing metacognitive awareness, decentralizing, and accepting their mental contents. This study aims to investigate the efficacy of mindfulness training in decreasing emotional schemas (rumination, not expressing emotions, lack of values and feeling guilty) in mothers with children with CP.

Materials \& Methods The present study is quasi-experiment with pretest-posttest and control group. The statistical population consists of all the mothers with CP children referring to the rehabilitation center of Mashhad City. The study sample consisted of 20 volunteers who were selected based on the results of their pretest and study criteria. They were selected and randomly put into control and experimental groups. The inclusion criteria included having at least a guidance education level to do better teaching assignments and having a child with $\mathrm{CP}$ whose severity of disorder was moderate or severe on the basis of a doctor's diagnosis. The study instrument was Leahy Emotional Schema Scale (LESS) which was answered by mothers in the control and experimental groups before the intervention. The participants in the experimental group received "Mindfulness Awareness" group intervention in eight 90-minute sessions for two months and the control group didn't receive any treatment.

Results The data were analyzed through Analysis of Covariance (ANCOVA) and t-test. The findings indicate that mindfulness training for mothers in the experimental group leads to a decrease in schemas like rumination $(t=2.57 ; \mathrm{P}<0.05)$, not expressing emotions $(\mathrm{t}=8.87 ; \mathrm{P}<0.05)$ and feeling guilty $(\mathrm{t}=2.4 ; \mathrm{P}<0.05)$, but it doesn't have a significant effect on the lack of values ( $F=2.73 ; P>0.05)$.

Conclusion Mindfulness can improve emotional schemas in mothers who have children with $\mathrm{CP}$ through raising awareness and concentration as effective training. Mindfulness education provides a model for reducing rumination and educates mothers to be aware of their mood fluctuations and to use mind-alert techniques to understand the information processing that makes emotions keep obsessing. Increasing calmness and consciousness along with a kind-hearted behavior creates curiosity and satisfaction at the present, which results in decreased rumination. The higher values in mothers are unchangeable beliefs, which are the function of culture and are unique to each individual. Therefore, the resistance derived from beliefs as a result of the education of mindfulness has a negative effect on the concept of higher-value inferiority. In sitting meditation, one can allow herself to express excitement with mercilessness and curiosity. Then he or she will be conscious of the gesture and state of the face and adapting it to its current state, and express her or his emotions and physical feelings without suppression and change. Feeling self-compassion in the mind of consciousness accepts the suffering of sin as part of life and leads to motivating the individual to reduce pain and suffering and feelings of guilt.

\section{* Corresponding Author:}

Ali Akbar Soleimanian, PhD.

Address: Department of Counseling, Guidance and Education, Faculty of Humanities, University of Bojnord, Bojnord, Iran.

Tel: +98 (915) 5844357

E-Mail: soliemanian@gmail.com 


\title{
اثربخشى آموزش ذهن آكاهى بر طرحوارههاى هيجانى ناساز تار مادران كودكان با فلج مغزى
}

\author{
نسيم اقدسى' هـ، "علىاكبر سليمانيان' ــ، رقيه اسدى كندمانى' \\ 1- كروه مشاوره، راهنماييى و علوم تربيتى، دانشكده علوم انسانى، دانشكاه بجنورده، بجنورده، ايران.

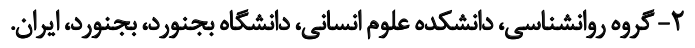

\begin{abstract}
حكبن

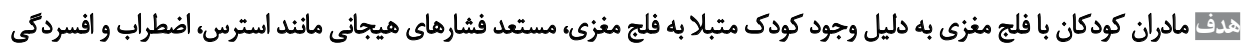

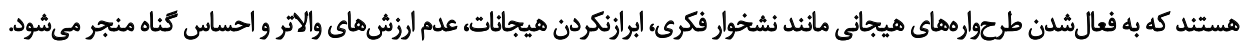

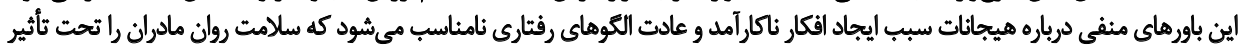

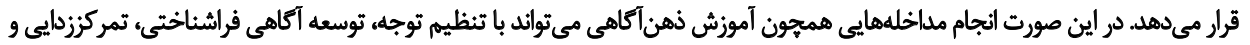

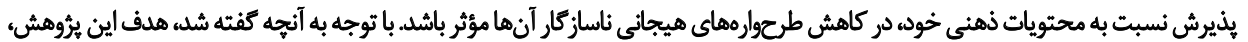

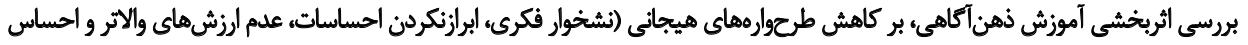

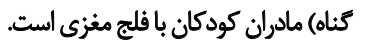

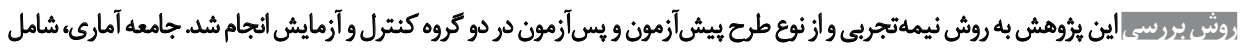

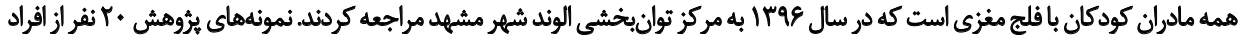

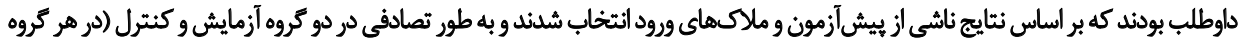

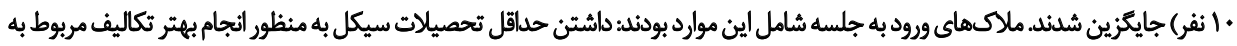

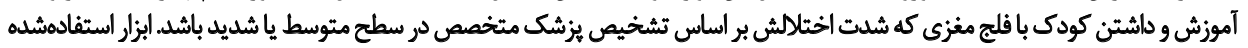

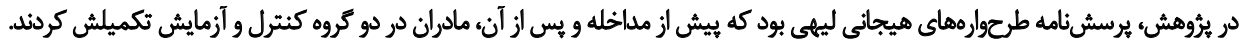

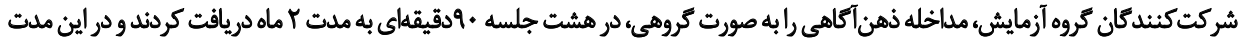

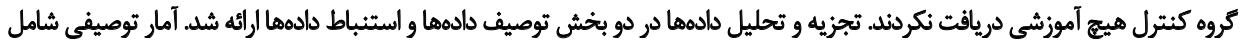

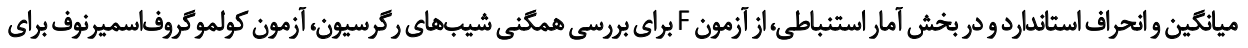

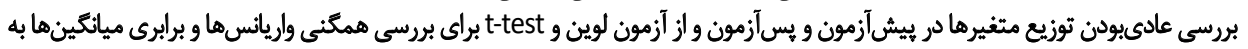

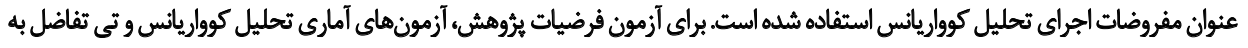

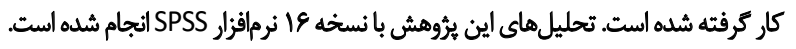

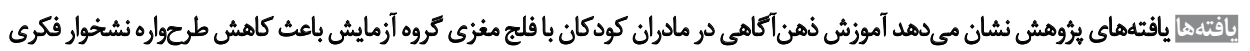

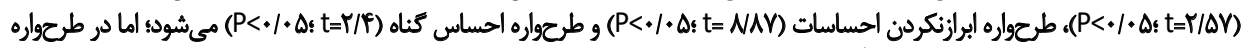

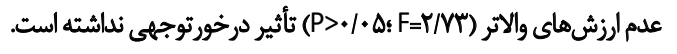

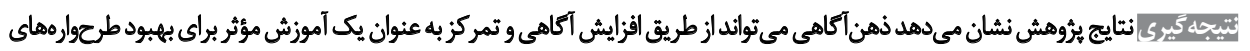

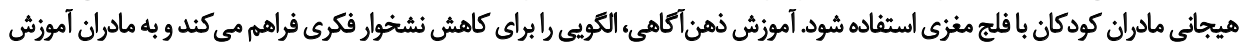

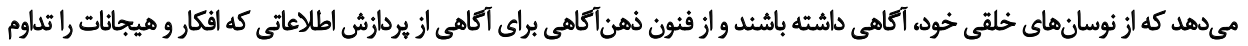

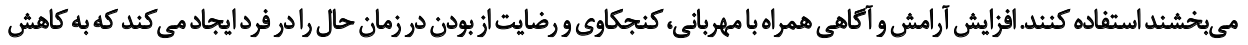

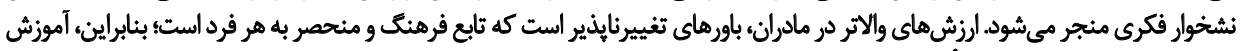

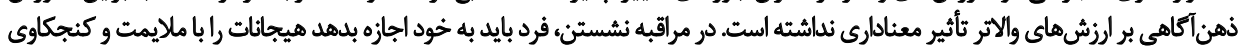

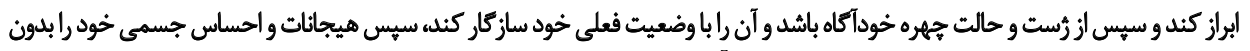

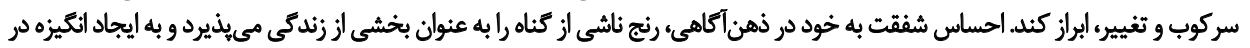

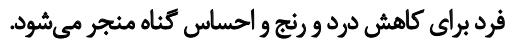

تاريخ دريافت: 9. شهريور بو

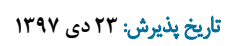

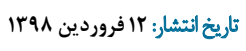

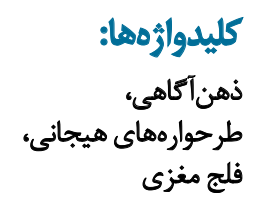

فلج مغزى حورئ 
مي شود. موارد ذكرشده نشاندهنده شيوههايى از ارزيابى فرد

مقلمه

نسبت به هيجانات منفى خود است [rهارد

نكرش و باورهاي منفى نسبت به هيجانات يا به عبارت ديكر،

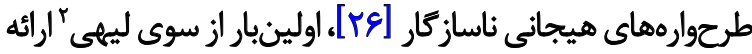

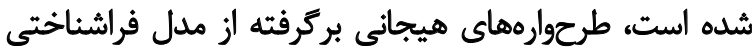

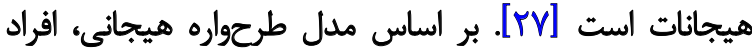

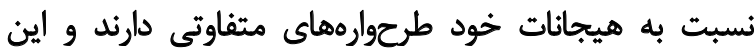

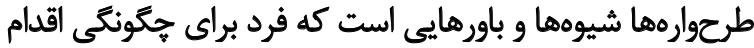

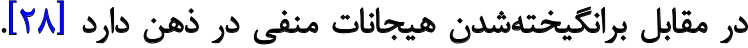

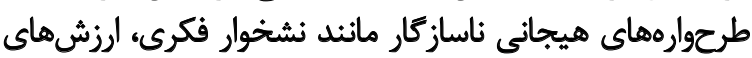

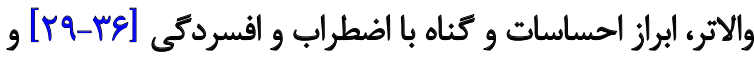

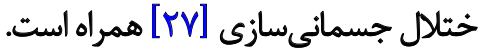

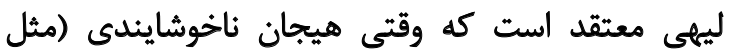

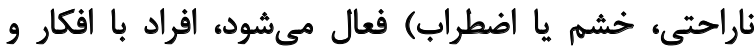

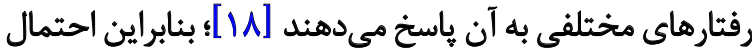

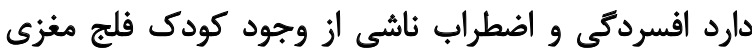

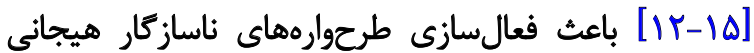

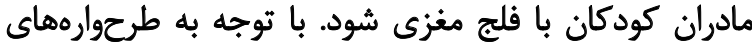

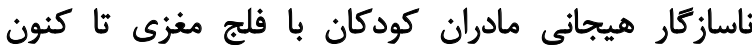

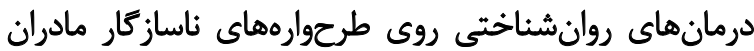

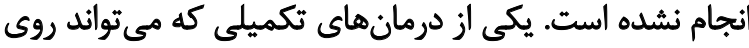

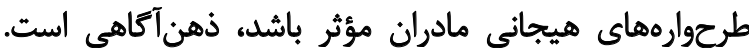

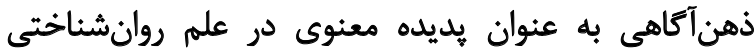

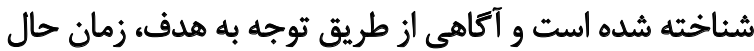

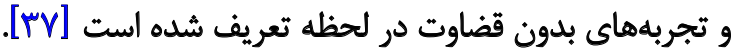

ذهن آكاهي باعث افزايش آتاهى، تمركززدايي، اجازه ظهور

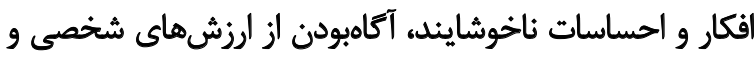

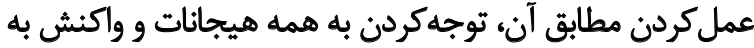

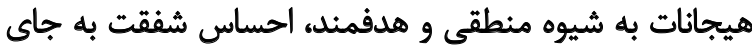

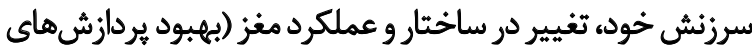
شناختى و هيجانى) و تقويت توجه و و حافظه فعال (بهبيود كاركرد

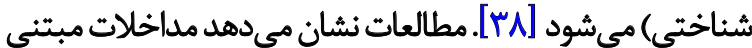

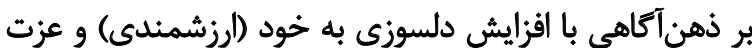

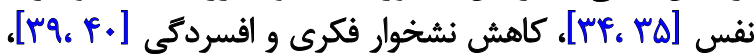

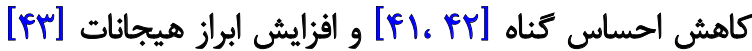

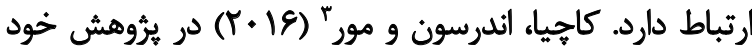

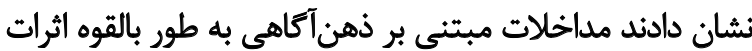

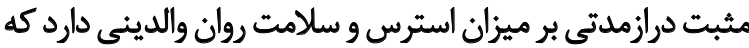

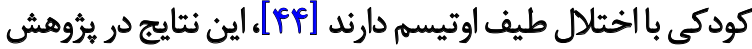

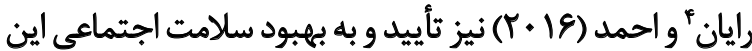

2. Leahy

3. Cachia, Anderson \& Moore

4. Rayan

1. Cerebral Palsy

فلج مغزى' بيمارى نورولوريكى است كه به دليل آسيب به فيه

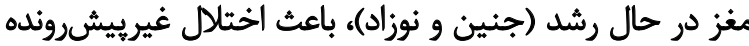

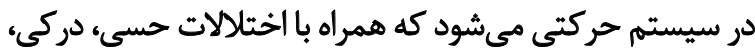

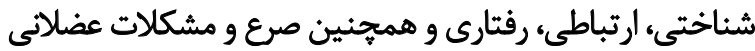

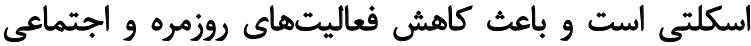

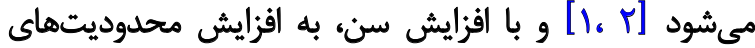

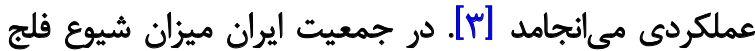

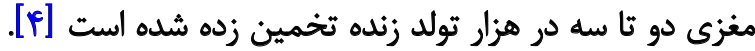

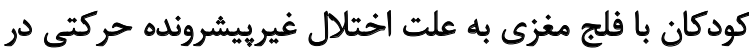

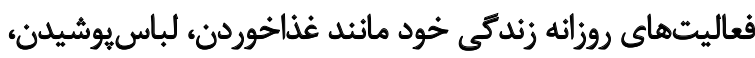

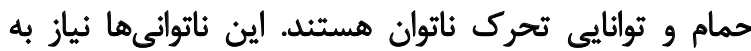

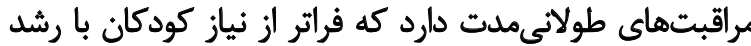

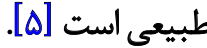

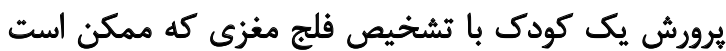

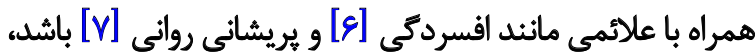

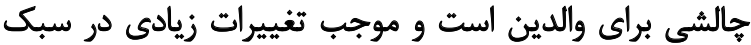

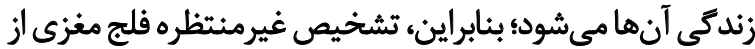

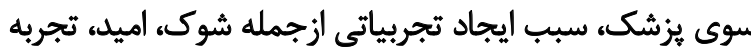

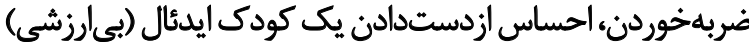

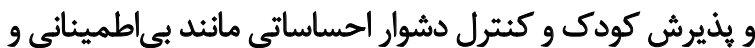

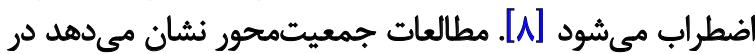

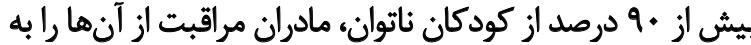

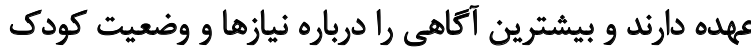

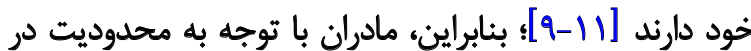

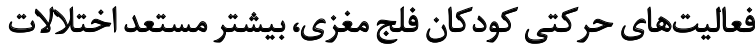

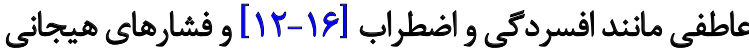

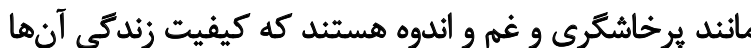

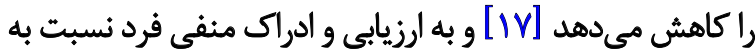

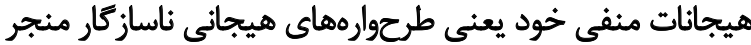

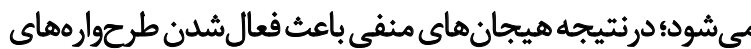

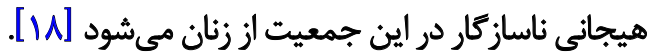

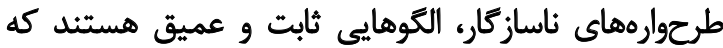

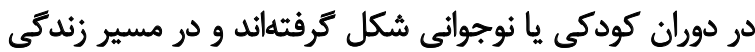

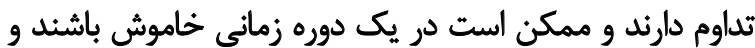

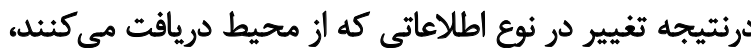

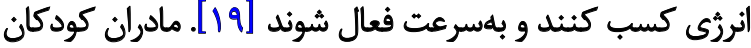

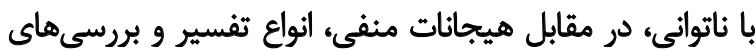

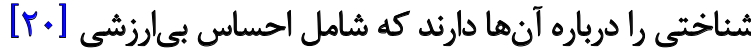

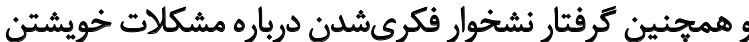

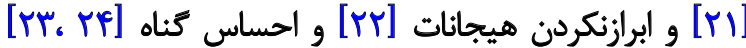


جدول ا. خلاصه جلسات آموزش ذهن آكاهى

\begin{tabular}{|c|c|}
\hline برنامه & جلسات/موضوع \\
\hline 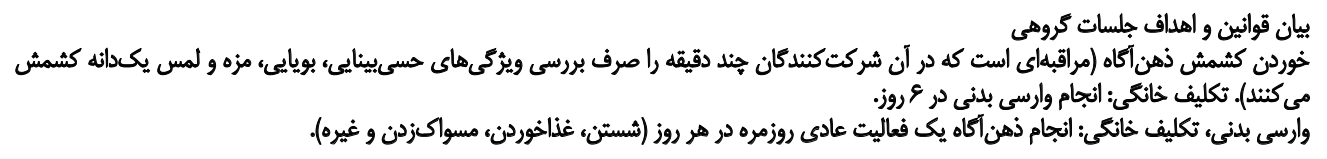 & جلسه الول/ / هدايت \\
\hline مرور تكاليف هفته كذشته، تمرين افكار و احساسات، تكليف خانكى: ثبت وقايع خوشايند و ناخوشايند & جلبه دوم/ بورياروييع \\
\hline 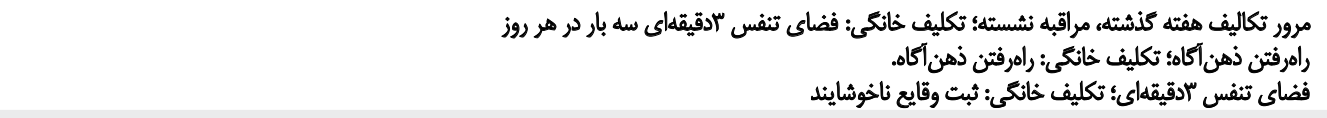 & جلسه سوم/ حضور ذهن \\
\hline 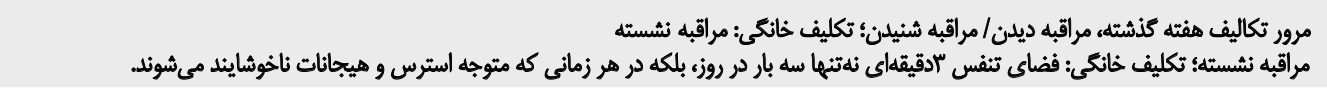 & 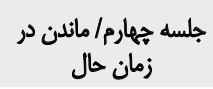 \\
\hline مرور تكاليف هفته كنشته، مراقبه نشستن: تكليف خانكى: مراقبه نشسته & جلسه ينجم/ اجازه و \\
\hline 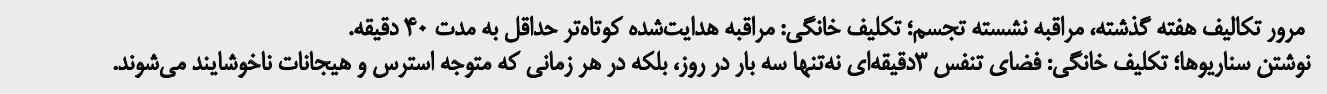 & جلسه ششم/افكار \\
\hline 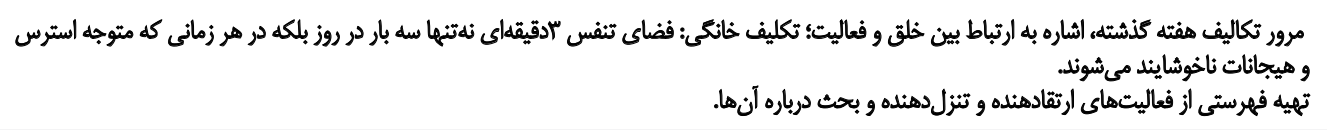 & جلسه هفتم/ مراقبت \\
\hline 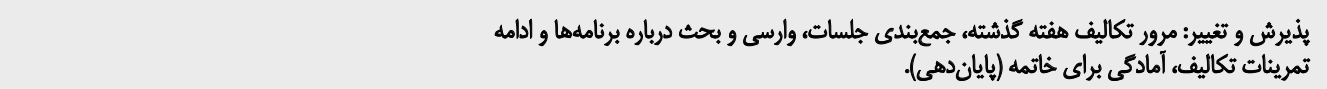 & جلسه هشتم/الستفاده ازز \\
\hline
\end{tabular}

يرؤهش شامل همه مادران كودكان فلج مغزى مركز توانبخشى

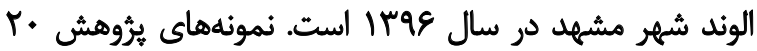

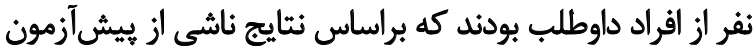

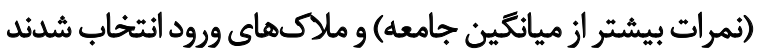

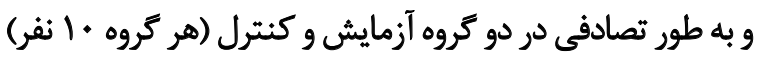

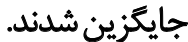

از جمله ملاكهاي ورود مي توان به اين موارد اشاره كرد: به جلسه داشتن حداقل تحصيلات سيكل به منظور انجام بهيتر

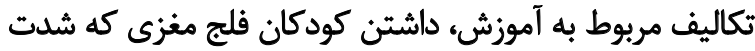

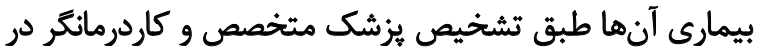

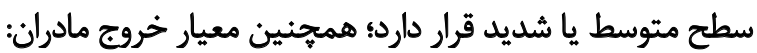

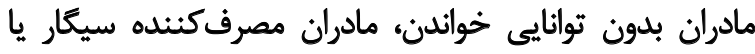

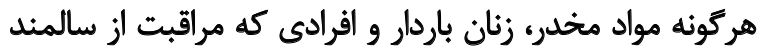

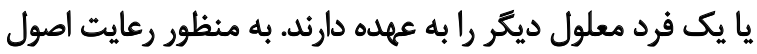
اخلاقى در يرسشنامه، به محرمانهبودن اطلاعات اشاره شد.

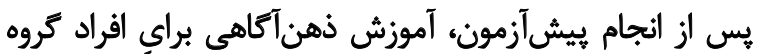

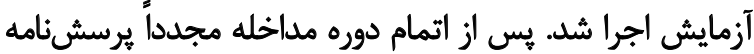

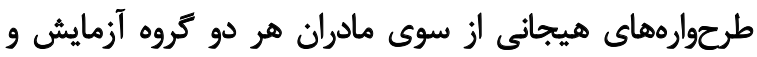

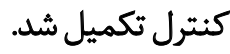

گيس از يايان جمعآورى داده بر اساس اصول اخلاقي، مداخله

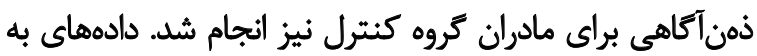

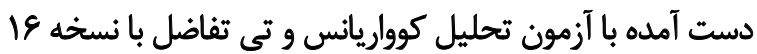

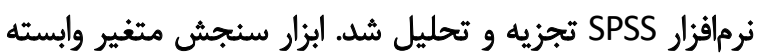

والدين منجر شد [Fه]]. نتايج يروهش هائا حاكى از اثرات مثبت آموزش ذهن آكاهي بر ناراحتى هاي روان شيناختى مادران كود كان

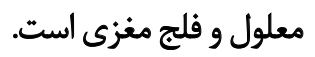

در مداخلات مبتنى بر ذهن آكاهى، به بيماران آموزش داده

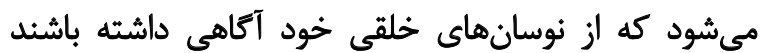

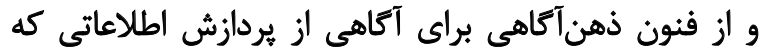

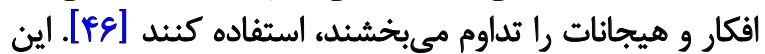

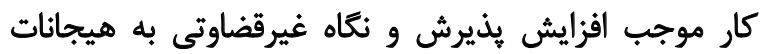

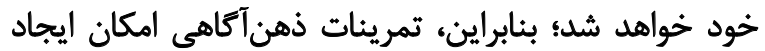

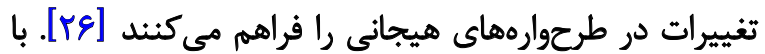

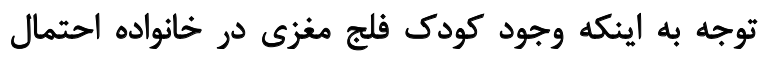
ايجاد طرحوارههاى هيجانى ناساز

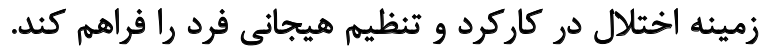

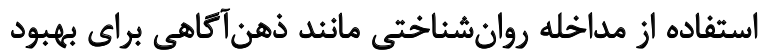

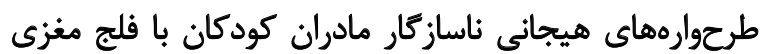

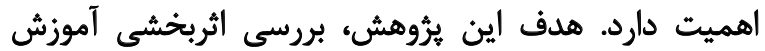

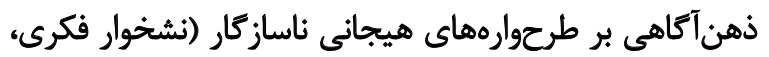

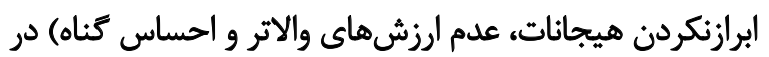
مادران كودكان با فلج مغزي است عدم ارتش

$$
\text { و وش بروسى }
$$

اين مطالعه به روش نيمهآزمايشى و از نوع طرح ييش آزمون

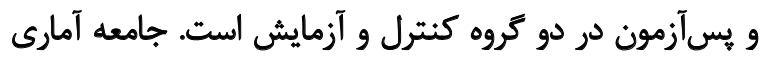


و عدم ارزشهاى والاتر) انتخاب شد. شركتكنيندكان مداخله

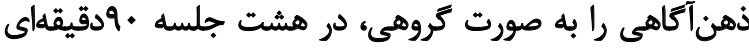

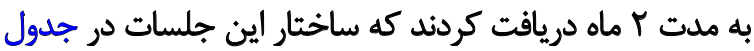
شماره آ آمده است دمبافت

ياقتثهها

نتايج حاصل از آمار توصيفى اين يُوهش به تفكيك گروهوهاى

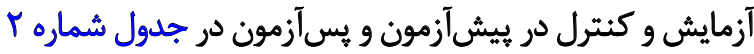

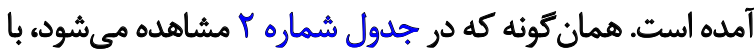

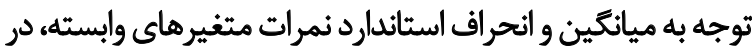
كروه آزمايش تغييرات بيشترى ديده مي مئنود. با توجه به نتايج آزمون كلموكروفاسميرنوف، براى بررسى

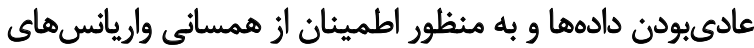

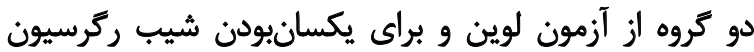

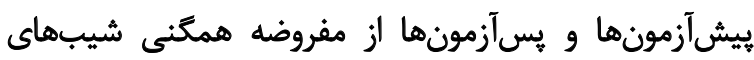

در اين يرؤشش، يرسشنامه طرحوارههاى هيجانىه است. اين

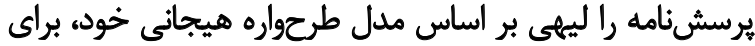

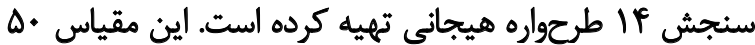

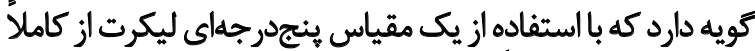

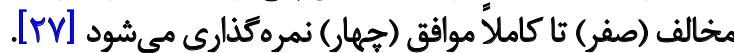

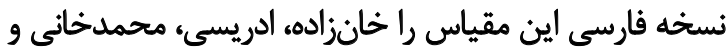

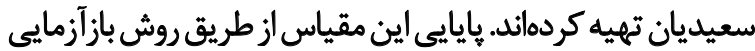

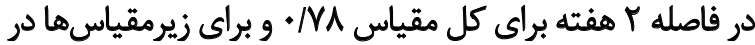
دامنهاي بين

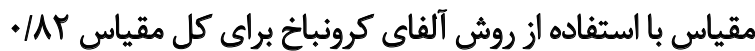

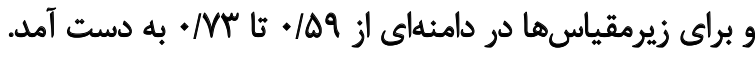

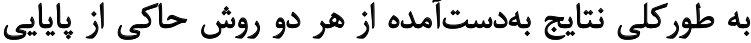

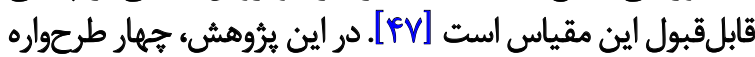
هيجانى (نشخوارفكرى، ابرازنكردن احساسات، احساس كناس

5. Leahy emotional shema scale (LESS)

جدول Y. ميانعين و انحراف اسثاندارد متغيرهاى بيروهش به تفكيك كروهها در بيش آزمون و يسآزمون

\begin{tabular}{|c|c|c|c|c|c|}
\hline \multicolumn{2}{|c|}{ بس آزمون } & \multicolumn{2}{|c|}{ يشىآزمون } & \multirow{2}{*}{ كروه } & \multirow{2}{*}{ مؤلثه } \\
\hline انحراف استاندارد & مياتكين & انحراف استاندارد & مياتكين & & \\
\hline$r / r q$ & s/A. & T/AV & $9 / \Delta$. & أزمايش & \multirow[b]{2}{*}{ نشخوار فكرى } \\
\hline$r /+H^{+}$ & e/f. & $r / M^{C}$ & 8 & كتترل & \\
\hline$V / \Delta E$ & $\mathbb{W} /$ & $W$ & $r / \%$. & آزمايش & \multirow{2}{*}{ عدم الرزش هاى والاتر } \\
\hline $1 / M$ & $r / \pi$ & $1 / 1$. & r & كنترل & \\
\hline.$/ 4 V$ & 1 &.$N$ & $V / \Delta$. & كنترل & \multirow{2}{*}{ برازتكردن احساسات } \\
\hline 1/9 & $r / A$. & $t / R$ & p & آزمايش & \\
\hline$T / T r$ & r/s. & $r / R^{T}$ & $N \Delta$. & كثترل & \multirow{2}{*}{ احساس كناه } \\
\hline $1 / 19$ & $\Delta / f$. & T/AS & $9 / \lambda$. & أزمايش & \\
\hline
\end{tabular}

جدول ب. بررسى عادىبودن توزيع نمرات تفاضلى

\begin{tabular}{|c|c|c|c|}
\hline مقدار احتمال & آماره z كلموكروفاسميرنوف & تروه & مؤلفه \\
\hline$\cdot M^{2}$ & . IFA & أزمايش & \multirow{3}{*}{ نشخوار فكرى } \\
\hline & & & \\
\hline$\cdot|k|$ & $\cdot / M$ & كتترل & \\
\hline.$/ 1 F$ & I/M & 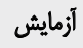 & \multirow{2}{*}{ برازنكردن احساسات } \\
\hline H &.$/ 81$ & كنترل & \\
\hline$\cdot M$ & $.19 V$ & أزمايش & \multirow{2}{*}{ احساس كناه } \\
\hline$\cdot M$ & . $k 9$ & كتترل & \\
\hline
\end{tabular}


جدول †. نتيجه تحليل كوواريانس عدم ارزشهاي والاتر در كروههاى آزمايش و كنترل

\begin{tabular}{|c|c|c|c|c|c|c|c|}
\hline ضريب اتا & مقدار احتمال & $\mathbf{F}$ & ميانكين مجذورات & درجه آزادى & مجموع مجذورات & منيع & متغير \\
\hline & .1 .9 & $r / \pi r$ & NEP & 1 & NEP & ييش آزمون & \\
\hline . &.$M$ & $T / M$ & $V / m 1$ & 1 & $V(r)$ & مداخله كروه & علم ارزش هاى والاتي \\
\hline - & - & - & r/gA & iv & $P \Delta / \Delta \Delta$ & خطا & \\
\hline
\end{tabular}

جدول هـ مقايسه ميانكين نمرات تفاضل دو كروه در مؤلفهها.

\begin{tabular}{|c|c|c|c|c|c|c|}
\hline \multicolumn{4}{|c|}{ أزمون t براى برابرى مياتكينها } & \multicolumn{2}{|c|}{ آزمون لوين } & \multirow{2}{*}{ مؤلفه } \\
\hline اختلاف ميانكين دو كروه & مقتدار & درجه آزادى & $\mathrm{t}$ & مقدار احتمال & F لوين F لو F & \\
\hline r/. & .1 .1 & in & T/AY &.$/ T Y$ & $1 / r \Delta$ & نشخُوارفكرى \\
\hline$\Delta / r *$ & $\%$ & IV/AS & NAY &.$/ r$ & $g / 9 Y$ & عدم ابرازنكردن احساسات \\
\hline$r / \Delta$. & $.1 . r$ & IA & $r / P$. &.$/ A P$ &.$/ \% A$ & الجساس كناه \\
\hline
\end{tabular}

توانبخننى

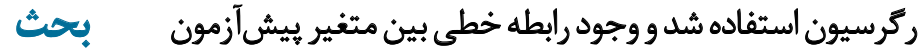

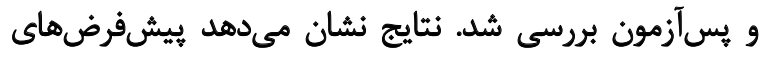
هدف از اين بثروهش، بررسى اثربخشى آموزش ذهن آناهي بر

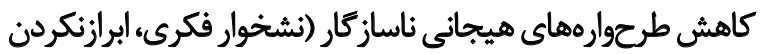

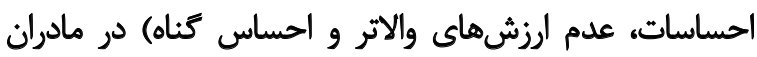

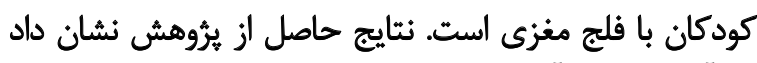

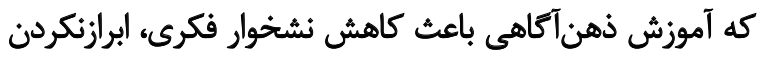

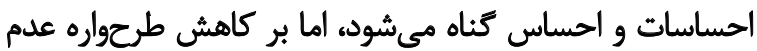
ارزش هاى والاتر اثركذار نيست.

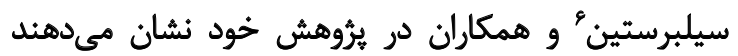

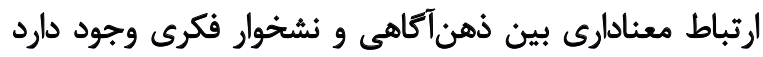

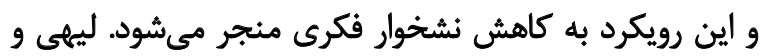

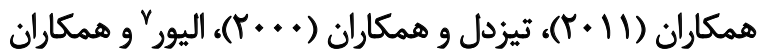
(Y. T)

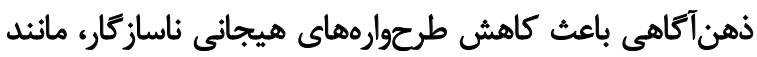

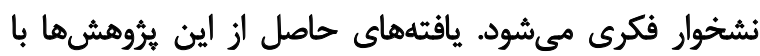

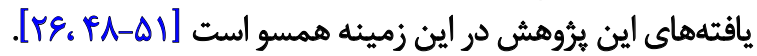
در تبيين نتايج بهدست آمده مى توان كفت آموزش ذهن في آكاهي،

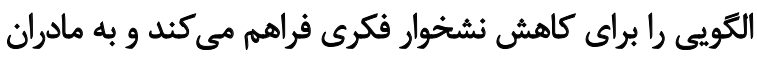

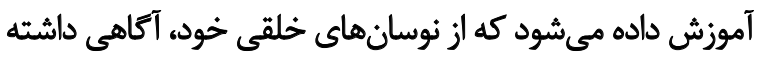

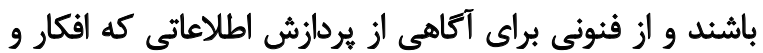

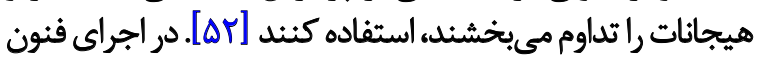

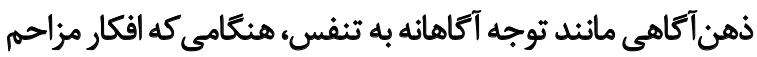

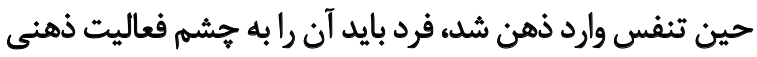

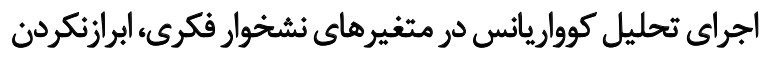

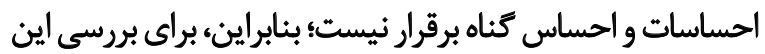

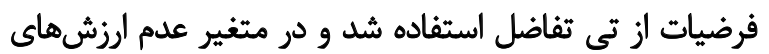

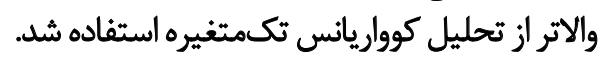
با توجه به جدول شمون شماره با، مقادير احتمال آزمون

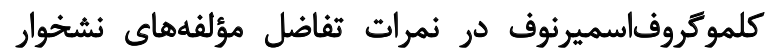

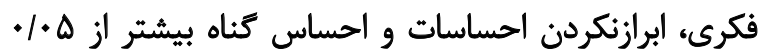

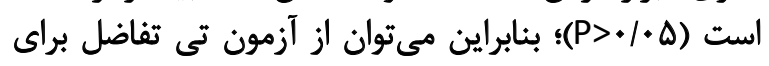

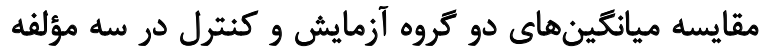

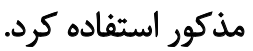
با توجه به نتايج جدول شماره f أل، بين افراد حاضر در كروهمهاى

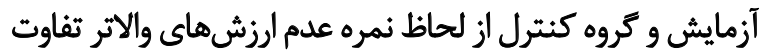

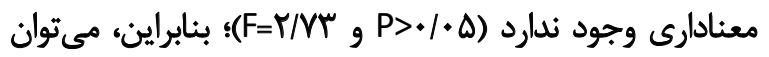

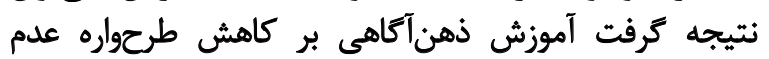

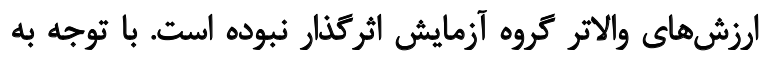

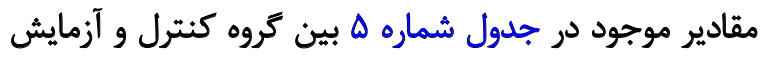

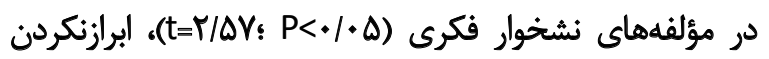
احساسات (t=r/F

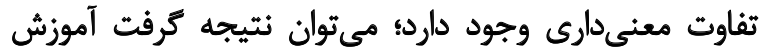

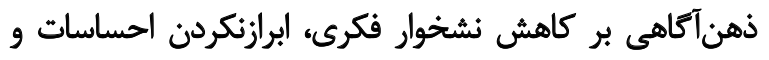

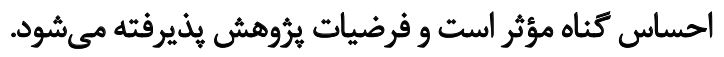


در تبيين يافتهها ميتوان بيان كرد كه در فنون ذهن آكاهي

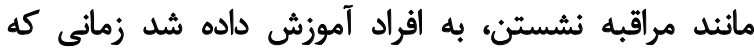

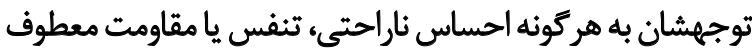

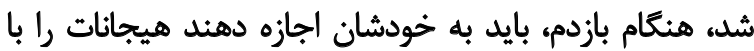

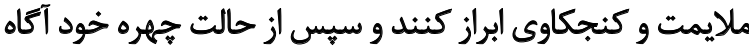

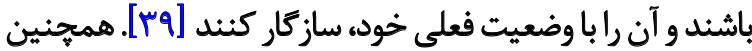

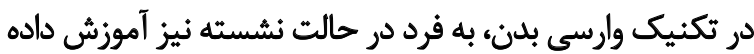

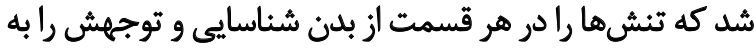

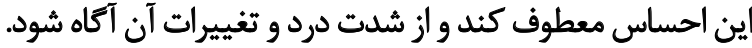

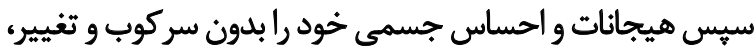

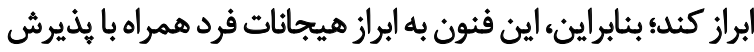

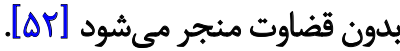

يافتههاى حاصل از اين يُروهش با يافتههاى بتمز 'و وهمكاران

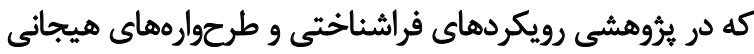

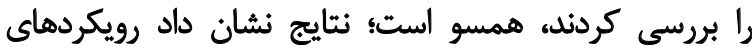

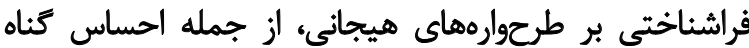

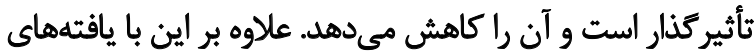

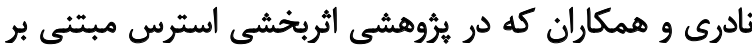

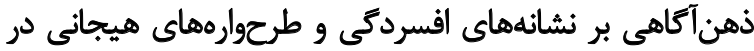

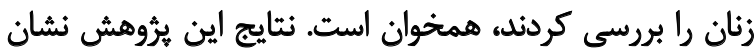

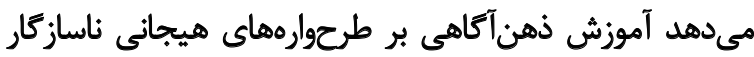

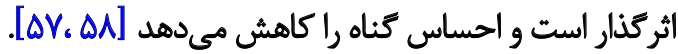

در تبيين اين فرضيه مىتوان كفت تكنيكهاى ذهن آكاهيى

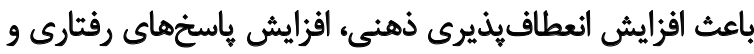

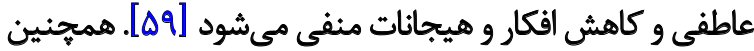

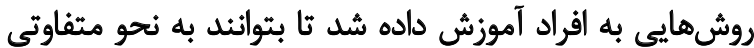

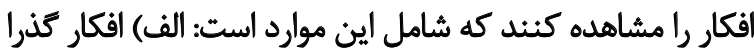

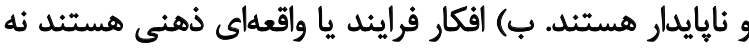

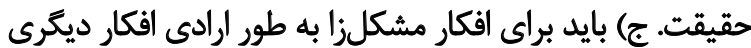

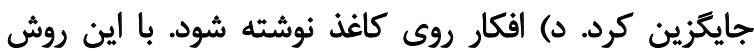

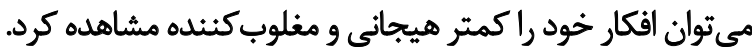

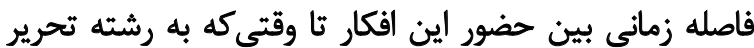

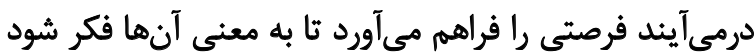

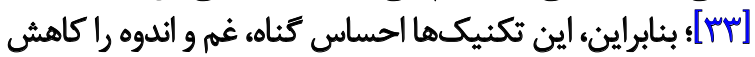

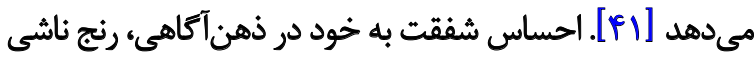

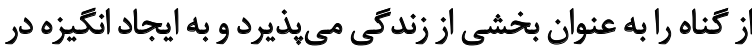

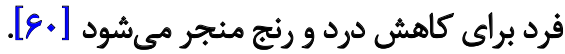

تكنيك شفقت به خود شامل سه بخش است: ا. آكاهي ذهنى

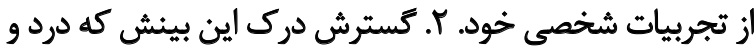

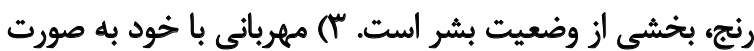

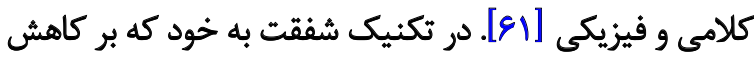

10. Batmaz, Kaymak, Kocbiyik \& Turkcapar
مشاهده كند و به عنوان نظارهكر، به افكارى كه با احساسات

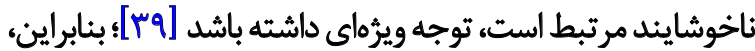

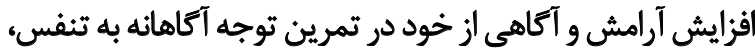

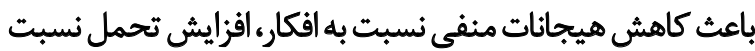

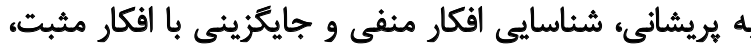

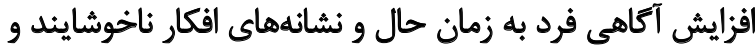

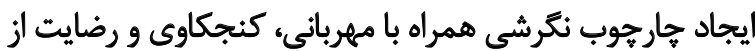

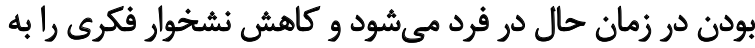

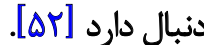

در تمرين كشمشخوردن، آزمودنىها بيى مى مبرند تجربهاى

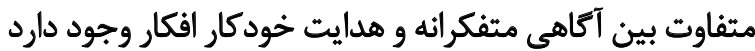

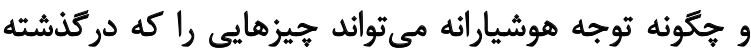

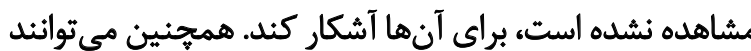

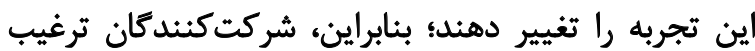

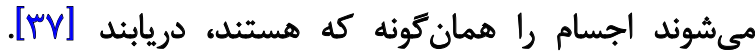

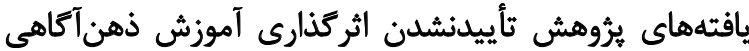

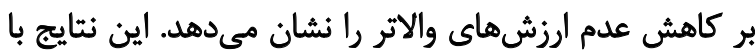

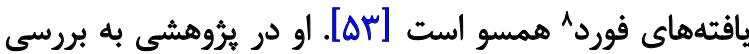

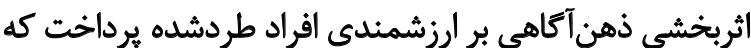

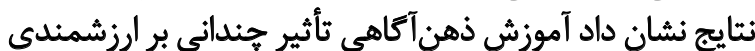
افراد طردشده ندارد [بهان]. ارزشها، عقايد بايدار و تغييرنايذير فرد نسبت به شيوه خاص

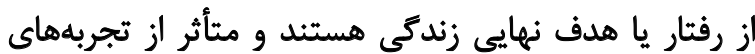

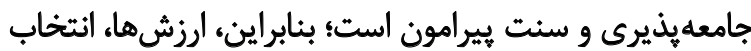

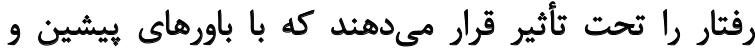

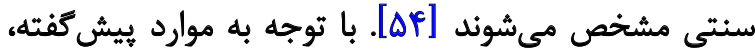

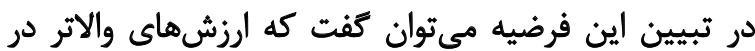

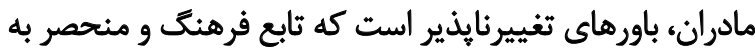

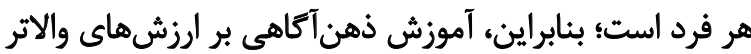
تأثير معنادارى نداشته است

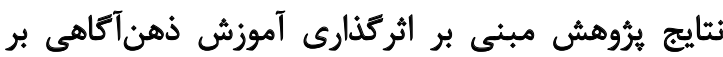

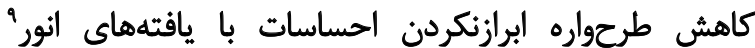

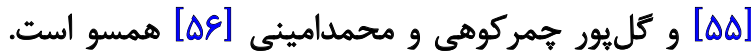

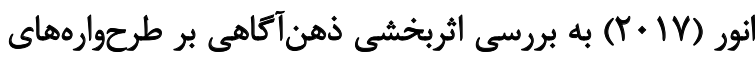

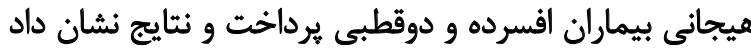

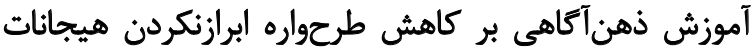

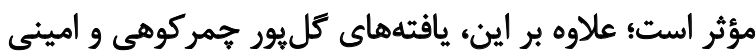

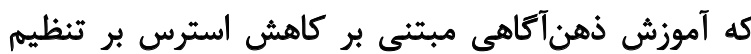

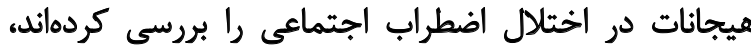

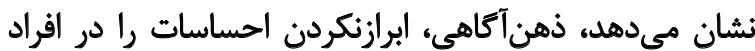

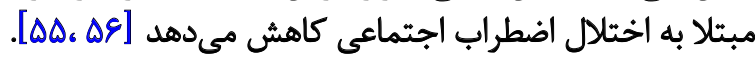


اينكه يّ از يايان جمعآورى دادهاء مداخله ذهن آناهي براى اعضاى كروه كنترل نيز اجراشد.

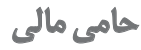

اين مقاله، مستخرج از يايان نامه كارشناسىارشد نويسنده اول

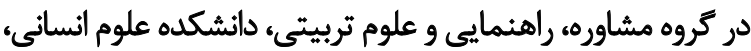
دانشكاه بجنورد است.

$$
\text { مشاركت نويسند مَّان }
$$

مشاركت نويسندكان به شرح زير است: مفهومسازى، تحقيق

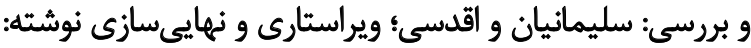
سليمانيان، اقدسى و اسدى كندماني.

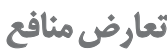

بنابر اظهار نويسندكان، اين مقاله تعارض منافع ندارد.
احساس كناه مؤثر بود، از افراد خواسته شد فعاليت لذتبخششى را

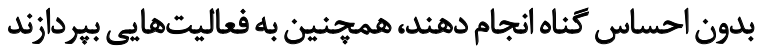

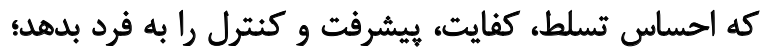

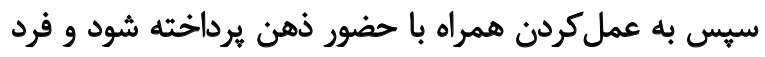

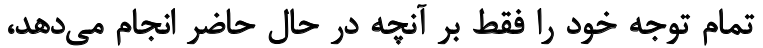

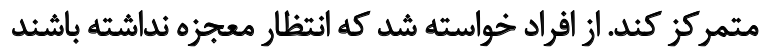

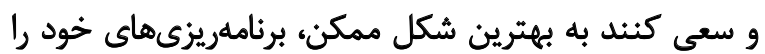

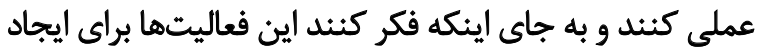

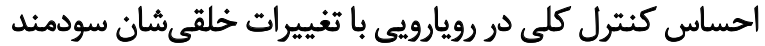

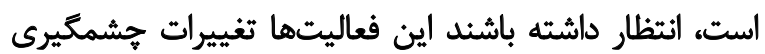

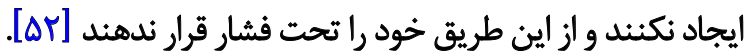

\section{نتيجلئيرى}

با توجه به نتايج بهدستآمده، آموزش ذهن آكاهى باعث كاهش نشخوار فكرى، ابرازنكردن احساسات و احساس كأس كناه

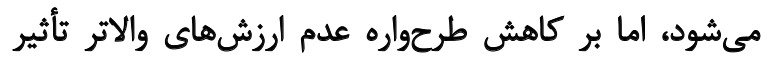

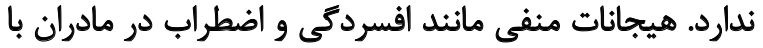

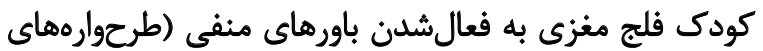

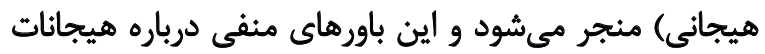

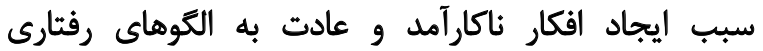

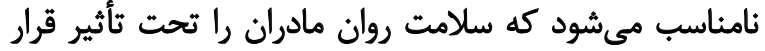

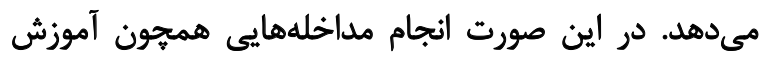

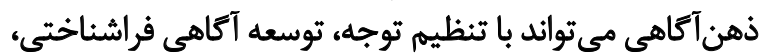

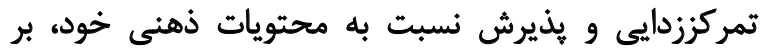

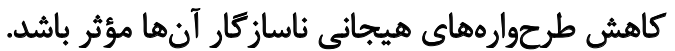

با توجه به محدوديتهاى يُؤوهش شامل نمونه ئيرى

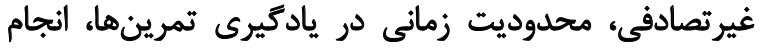

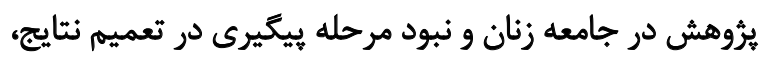

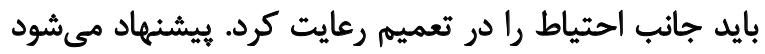

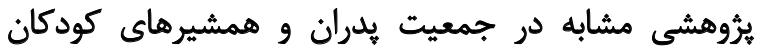

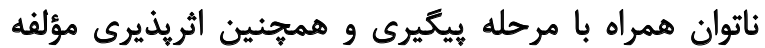

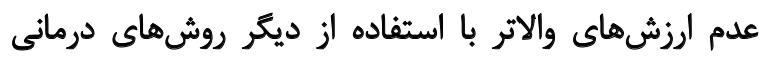

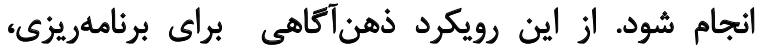

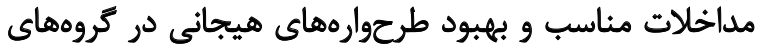

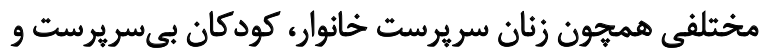
بدسريرست استفاده شود.

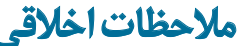

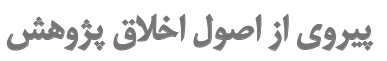

جهت رعايت اصول اخلاقى شركت كنندكان آكاهى كامل از إز

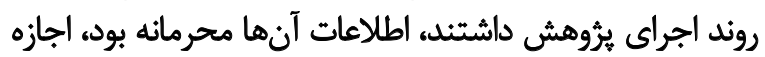

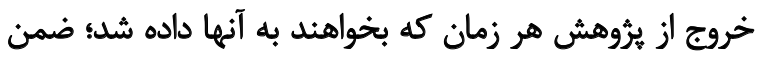




\section{References}

[1] Prudente COM, Barbosa MA, Porto CC. Relation between quality of life of mothers of children with cerebral palsy and the children's motor functioning, after ten months of rehabilitation. Revista Latino-Americana de Enfermagem. 2010 18(2):149-55. [DOI:10.1590/S0104-11692010000200002]

[2] Webb W, Adler RK. Neurology for the Speech-Language Pathologist-E-Book. Amsterdam: Elsevier Health Sciences; 2016.

[3] Razaviafzal Z. [A survey on knowledge and application of Iranian caregivers regarding special care of children 1-5 years old with cerebral palsy (Persian)] [MSc. thesis]. Tehran: University of Social Welfare and Rehabilitation Sciences; 2011.

[4] Soleimani F, Vameghi R, Hemmati S, Biglarian A, Sourtiji H. [Survey on types and associated disorders of cerebral palsy in eastern and northern districts of Tehran (Persian)]. Archives of Rehabilitation. 2011; 12(3):75-82.

[5] Altindag Ö, Işcan A, Akcan S, Koksal S, Erçin M, Ege L. Anxiety and depression levels in mothers of children with Cerebral Palsy. Turkish Journal of Physical Medicine \& Rehabilitation. 2007; 53(1):22-4.

[6] Sharifian-Sani M, Sajjadi H, Tolouei F, Kazem-Nezhad A. [Girls and women with physical disabilities: Needs and problems (Persian)]. Archives of Rehabilitation. 2006; 7(2):41-8.

[7] Dadkhah A. [The effect of psychorehabilitation program on children with physical and mental disability (Persian)]. Archives of Rehabilitation. 2007; 8(2):57-62.

[8] Fernández-Alcántara M, García-Caro MP, Laynez-Rubio $C$, Pérez-Marfil MN, Martí-García C, Benítez-Feliponi Á, et al. Feelings of loss in parents of children with infantile cerebral palsy. Disability and Health Journal. 2015; 8(1):93-101. [DOI:10.1016/j.dhjo.2014.06.003] [PMID]

[9] Brehaut JC, Kohen DE, Raina P, Walter SD, Russell DJ, Swinton $M$, et al. The health of primary caregivers of children with cerebral palsy: How does it compare with that of other Canadian caregivers? Pediatrics. 2004; 114(2):e182-e91. [DOI:10.1542/ peds.114.2.e182] [PMID]

[10] Nobakht Z, Rassafiani, M. [Review of caring training for caregivers of children with cerebral palsy (Persian)]. Journal of Rehabilitation Medicine. 2016; 5(3):175-83.

[11] Montes G, Halterman JS. Psychological functioning and coping among mothers of children with autism: A populationbased study. Pediatrics. 2007; 119(5):e1040-e6. [DOI:10.1542/ peds.2006-2819] [PMID]

[12] Dave D, Mittal S, Tiwari D, Parmar M, Gedan S, Patel V. Study of anxiety and depression in caregivers of intellectually disabled children. Journal of Research in Medical and Dental Science. 2017; 2(1):8-13. [DOI:10.5455/jrmds.2014212]

[13] Garip Y, Ozel S, Tuncer OB, Kilinc G, Seckin F, Arasil T. Fatigue in the mothers of children with cerebral palsy. Disability and Rehabilitation. 2017; 39(8):757-62. [DOI:10.3109/09638288.2 016.1161837] [PMID]

[14] Kumar R, Lakhiar MA, Lakhair MA. Frequency and severity of depression in mothers of cerebral palsy children. Journal of Liaquat University of Medical and Health Sciences. 2016; 15(3):147-51.

[15] Toulgui E, Jemni S, Samia F, Lazreg N, Mtaouaa S, Khachnaou F. Depression and anxiety in mothers of children with cerebral palsy: Comparative study. Annals of Physical and Rehabilitation Medicine. 2016; 59(Suppl):e9. [DOI:10.1016/j. rehab.2016.07.024]

[16] Ahmadizadeh Z, Mokhlesin M. Factors Which Affect the Depression of Mothers with Cerebral Palsy Child. Iranian Rehabilitation Journal. 2014; 12(4):43-8.

[17] Yun CK. Relationship between the quality of life of the caregiver and motor function of children with cerebral palsy. Physical Therapy Rehabilitation Science. 2017; 6(1):26-32. [DOI:10.14474/ptrs.2017.6.1.26]

[18] Leahy RL. Emotional schema therapy. New York: Guilford Publications; 2015.

[19] Martin R, Young J. Schema therapy. In: Dobson K, editor Handbook of Cognitive-Behavioral Therapies. New York: Guilford Publications; 2010.

[20] Findler L, Jacoby AK, Gabis L. Subjective happiness among mothers of children with disabilities: The role of stress, attachment, guilt and social support. Research in Developmental Disabilities. 2016; 55:44-54. [DOI:10.1016/j.ridd.2016.03.006] [PMID]

[21] Zhang W, Yan TT, Du YS, Liu XH. Relationship between coping, rumination and posttraumatic growth in mothers of children with autism spectrum disorders. Research in Autism Spectrum Disorders. 2013; 7(10):1204-10. [DOI:10.1016/j. rasd.2013.07.008]

[22] Taylor CT, Bomyea J, Amir N. Attentional bias away from positive social information mediates the link between social anxiety and anxiety vulnerability to a social stressor. Journal of Anxiety Disorders. 2010; 24(4):403-8. [DOI:10.1016/j. janxdis.2010.02.004] [PMID] [PMCID]

[23] Francis A. Stigma in an era of medicalisation and anxious parenting: How proximity and culpability shape middle-class parents' experiences of disgrace. Sociology of Health \& IIIness. 2012; 34(6):927-42. [DOI:10.1111/j.1467-9566.2011.01445.x] [PMID]

[24] Neff KD, Faso DJ. Self-compassion and well-being in parents of children with autism. Mindfulness. 2015; 6(4):938-47. [DOI:10.1007/s12671-014-0359-2]

[25] Leahy RL. Emotional schemas and resistance to change in anxiety disorders. Cognitive and Behavioral Practice. 2007; 14(1):36-45. [DOI:10.1016/j.cbpra.2006.08.001]

[26] Leahy RL, Tirch D, Napolitano LA. Emotion regulation in psychotherapy: A practitioner's guide. New York: Guilford Publications; 2011.

[27] Leahy RL. A model of emotional schemas. Cognitive and Behavioral Practice. 2002; 9(3):177-90. [DOI:10.1016/S10777229(02)80048-7]

[28] Andriopoulos P, Kafetsios K. Priming the secure attachment schema: effects on emotion information processing. Psihologijske Teme. 2015; 24(1):71-89. 
[29] Rueger SY, George R. Indirect effects of attributional style for positive events on depressive symptoms through self-esteem during early adolescence. Journal of Youth and Adolescence. 2017; 46(4):701-8. [DOI:10.1007/s10964-016-0530-2] [PMID]

[30] Orth U, Robins RW, Meier LL, Conger RD. Refining the vulnerability model of low self-esteem and depression: Disentangling the effects of genuine self-esteem and narcissism. Journal of Personality and Social Psychology. 2016; 110(1):133-149. [DOI:10.1037/pspp0000038] [PMID]

[31] Michl LC, McLaughlin KA, Shepherd K, Nolen-Hoeksema S. Rumination as a mechanism linking stressful life events to symptoms of depression and anxiety: Longitudinal evidence in early adolescents and adults. Journal of Abnormal Psychology. 2013; 122(2):339-52. [DOI:10.1037/a0031994] [PMID]

[32] Ruscio AM, Gentes EL, Jones JD, Hallion LS, Coleman ES Swendsen J. Rumination predicts heightened responding to stressful life events in major depressive disorder and generalized anxiety disorder. Journal of Abnormal Psychology. 2015; 124(1):17-26. [DOI:10.1037/abn0000025] [PMID] [PMCID]

[33] Hiller TS, Steffens MC, Ritter V, Stangier U. On the context dependency of implicit self-esteem in social anxiety disorder. Journal of Behavior Therapy and Experimental Psychiatry. 2017; 57:118-25. [DOI:10.1016/j.jbtep.2017.05.005] [PMID]

[34] Bajaj B, Robins RW, Pande N. Mediating role of self-esteem on the relationship between mindfulness, anxiety, and depression. Personality and Individual Differences. 2016; 96:127-31. [DOI:10.1016/j.paid.2016.02.085]

[35] Svendsen JL, Kvernenes KV, Wiker AS, Dundas I. Mechanisms of mindfulness: Rumination and self-compassion. Nordic Psychology. 2017; 69(2):71-82. [DOI:10.1080/19012276.2016.11 71730]

[36] Serbic D, Pincus T. The relationship between pain, disability, guilt and acceptance in low back pain: A mediation analysis. Journal of Behavioral Medicine. 2017; 40(4):651-8. [DOI:10.1007/s10865-017-9826-2] [PMID] [PMCID]

[37] Crane RS, Brewer J, Feldman C, Kabat-Zinn J, Santorelli S, Williams JMG, et al. What defines mindfulness-based programs? The warp and the weft. Psychological Medicine. 2017; 47(6):990-9. [DOI:10.1017/S0033291716003317] [PMID]

[38] Vreeswijk MV, Broersen J, Schurink G, Tempel JVD. Mindfulness and schema therapy. Hoboken, New Jersey: Wiley Blackwell; 2014. [DOI:10.1002/9781118753125]

[39] Paul NA, Stanton SJ, Greeson JM, Smoski MJ, Wang L. Psychological and neural mechanisms of trait mindfulness in reducing depression vulnerability. Social Cognitive and Affective Neuroscience. 2012; 8(1):56-64. [DOI:10.1093/scan/nss070] [PMID] [PMCID]

[40] Alsubaie M, Abbott R, Dunn B, Dickens C, Keil T, Henley W, et al. Mechanisms of action in Mindfulness-Based Cognitive Therapy (MBCT) and Mindfulness-Based Stress Reduction (MBSR) in people with physical and/or psychological conditions: A systematic review. Clinical Psychology Review. 2017; 55:74-91. [DOI:10.1016/j.cpr.2017.04.008] [PMID]
[41] Friese M, Hofmann W. State mindfulness, self-regulation, and emotional experience in everyday life. Motivation Science. 2016; 2(1):1-14. [DOI:10.1037/mot0000027]

[42] Held P, Owens GP, Monroe JR, Chard KM. Increased mindfulness skills as predictors of reduced trauma-related guilt in treatment-seeking veterans. Journal of Traumatic Stress. 2017; 30(4):425-31. [DOI:10.1002/jts.22209] [PMID]

[43] Brockman R, Ciarrochi J, Parker P, Kashdan T. Emotion regulation strategies in daily life: Mindfulness, cognitive reappraisa and emotion suppression. Cognitive Behaviour Therapy. 2017 46(2):91-113. [DOI:10.1080/16506073.2016.1218926] [PMID]

[44] Cachia RL, Anderson A, Moore DW. Mindfulness, stress and well-being in parents of children with autism spectrum disorder: A systematic review. Journal of Child and Family Studies. 2016; 25(1):1-14. [DOI:10.1007/s10826-015-0193-8]

[45] Rayan A, Ahmad M. Mindfulness and parenting distress among parents of children with disabilities: A literature review. Perspectives in Psychiatric Care. 2018; 54(2):324-30. [DOI:10.1111/ppc.12217] [PMID]

[46] Deen S, Sipe W, Eisendrath SJ. Mindfulness-based cognitive therapy for treatment-resistant depression. In: Eisendrath SJ, editor. Mindfulness-Based Cognitive Therapy. Berlin: Springer 2016.

[47] Mohammad Khani S, Khanzadeh M, Saeidian M. [Factor structure and psychometric characteristics on students' emotional Schemas scale (Persian)]. Journal of Clinical Psychology. 2013; 3(11):26-8.

[48] Silberstein LR, Tirch D, Leahy RL, McGinn L. Mindfulness, psychological flexibility and emotional schemas. International Journal of Cognitive Therapy. 2012; 5(4):406-19. [DOI:10.1521/ ijct.2012.5.4.406]

[49] Oliver JE, McLachlan K, Jose PE, Peters E. Predicting changes in delusional ideation: The role of mindfulness and negative schemas. Psychology and Psychotherapy: Theory, Research and Practice. 2012; 85(3):243-59. [DOI:10.1111/j.20448341.2011.02025.x] [PMID]

[50] Gojani PJ, Masjedi M, Khaleghipour S, Behzadi E. Effects of the schema therapy and mindfulness on the maladaptive schemas hold by the psoriasis patients with the psychopathology symptoms. Advanced Biomedical Research. 2017; 6:4 [DOI:10.4103/2277-9175.190988] [PMID]

[51] Teasdale JD, Segal ZV, Williams JMG, Ridgeway VA, Soulsby JM, Lau MA. Prevention of relapse/recurrence in major depression by mindfulness-based cognitive therapy. Journal of Consulting and Clinical Psychology. 2000; 68(4):615-23. [DOI:10.1037/0022-006X.68.4.615]

[52] Parsons CE, Crane C, Parsons LJ, Fjorback LO, Kuyken W. Home practice in Mindfulness-Based Cognitive Therapy and Mindfulness-Based Stress Reduction: A systematic review and meta-analysis of participants' mindfulness practice and its association with outcomes. Behaviour Research and Therapy. 2017; 95:29-41. [DOI:10.1016/j.brat.2017.05.004] [PMID] [PMCID]

[53] Ford MB. A Nuanced View of the Benefits of Mindfulness: Self-Esteem as a Moderator of the Effects of Mind- 
fulness on Responses to Social Rejection. Journal of Social and Clinical Psychology. 2017; 36(9):739-67. [DOI:10.1521/ jscp.2017.36.9.739]

[54] Wittgenstein L. Culture and value. Chicago: University of Chicago Press; 1984.

[55] Onur O. Emotional schemas: A new cognitive perspective for the distinction between unipolar depression and bipolar disorder. European Psychiatry. 2017; 41:S243. [DOI:10.1016/j. eurpsy.2017.02.016]

[56] Golpour CR, Mohammad AZ. [The efficacy of mindfulness based stress reduction on mindfulness and assertiveness of students with test anxiety (Persian)]. Journal of School Psychology. 2012; 1(3):82-100.

[57] Naderi Y, Parhoon H, Nouri R, Mohammadkhani S, Taghizade M. [Effect of Mindfulness-Based Stress Reduction (MBSR) on depression symptoms and emotional schema in women with major depressive disorder (Persian)]. Journal of Kermanshah University of Medical Sciences. 2015; 19(4):181-90.

[58] Batmaz S, Kaymak SU, Kocbiyik S, Turkcapar MH. Metacognitions and emotional schemas: A new cognitive perspective for the distinction between unipolar and bipolar depression. Comprehensive Psychiatry. 2014; 55(7):1546-55. [DOI:10.1016/j. comppsych.2014.05.016] [PMID]

[59] Crane R. Mindfulness-based cognitive therapy: Distinctive features. Abingdon: Taylor \& Francis; 2017. [DOI:10.4324/9781315627229]

[60] Gilbert P. Mindful compassion: Using the power of mindfulness and compassion to transform our lives. New York: Hachette; 2013.

[61] Neff KD. The development and validation of a scale to measure self-compassion. Self and Identity. 2003; 2(3):223-50. [DOI:10.1080/15298860309027] 
This Page Intentionally Left Blank 